\title{
Una aproximación a la identificación, medición y generación de los spillovers recíprocos*
}

\author{
An approach to identifying, measuring, and generating \\ reciprocal spillovers \\ Une approximation à l’identification, mesure et génération des \\ spillovers réciproques
}

Henry Caicedo Asprilla henry.caicedo@correounivalle.edu.co Universidad del Valle Cali - Colombia

Estudiante del Doctorado en Economía y Gestión de la Innovación, Universidad Autónoma de Madrid. Máster de Ciencias de la Organización, Universidad del Valle. Profesor de Comercio Internacional

y Economía del Cambio Tecnológico.

Artículo de revisión Según Clasificación Colciencias

Fecha de recepción: 06/03/2012

Fecha de corrección: $03 / 07 / 2012$

Fecha de aprobación: 04/12/2012

\section{Resumen}

En este documento se examina la literatura sobre los spillovers recíprocos, con el fin de contribuir a la comprensión que tienen estas externalidades en la organización industrial, la localización de las firmas multinacionales y la aglomeración de empresas. Pese a que la captura y representación de esta clase de externalidades ha sido objeto de críticas, aquí se recogen algunos trabajos que muestran la naturaleza e importancia de los spillovers recíprocos. Se aplicó el método bibliométrico de análisis de citas usando la base de datos Google Scholar. Se evidencia que los spillovers recíprocos se manifiestan a través de las citas de patentes, movilidad de la mano de obra entre empresas, el aprendizaje de formas de organización y producción, el compartimiento de canales de distribución, etc. De igual manera se muestra que los spillovers recíprocos surgen gracias a la configuración de redes entre las multinacionales y los actores de la región de llegada. Se concluye que si bien los spillovers recíprocos son difíciles de capturar y de medir, es posible seguir el rastro a este tipo de externalidades cuando el análisis se realiza en función del proceso de transferencia de tecnología.

Palabras clave: spillovers, transferencia de tecnología, redes de innovación, sistemas regionales de innovación, empresas multinacionales.

* Este artículo es un producto derivado del proyecto: "Una evaluación y comparación del papel de los sistemas de innovación en la jerarquía de los territorios de las ciudades región globales“, con código 8100, aprobado en la Convocatoria Interna 2-2012 para la Conformación de un Banco de Proyectos de Investigación de la Universidad del Valle. 
An approach to identifying, measuring, and generating reciprocal spillovers

\section{Una aproximación a la identificación, medición y generación de los spillovers recíprocos}

\section{Une approximation à l'identification, mesure et génération des spillovers réciproques}

\section{Abstract}

This paper reviews the literature on reciprocal spillovers to contribute to understanding these externalities in industrial organizations, location of multinational firms, and agglomeration of firms. Although the capture and representation of such externalities has been criticized, some work is presented herein showing the nature and importance of reciprocal spillovers. The method applied was bibliometric citation analysis using the Google Scholar database. Evidence exists that reciprocal spillovers manifest through patent citations, mobility of labor among firms, learning forms of organizations and production, magazine distribution channels, etc. It is, likewise, shown that reciprocal spillovers arise due to network configuration among multinational firms and the arrival of regional players. We conclude that while reciprocal spillovers are difficult to capture and measure, when the analysis is based on the technology transfer process, it is possible to trace these types of externalities.

Keywords: spillovers, technology transfer, innovation networks, Regional innovation Systems, Multinational firms.
Une approximation à l'identification, mesure et génération des spillovers réciproques

Una aproximación a la identificación, medición y generación de los spillovers recíprocos

An approach to identifying, measuring, and generating reciprocal spillovers

\section{Résumée}

Sur ce document on examine la littérature sur les spillovers réciproques, pour contribuer à la compréhension de ces externalités à l'intérieur de l'organisation industrielle, à la localisation des entreprises internationales et à l'agglomération d'entreprises. Si bien que la capture et la représentation de ce type d'externalités a été objet de critiques, on recueille ici quelques travaux qui démontrent la nature et l'importance des spillovers réciproques. On a appliqué la méthode bibliométrique d'analyse de citations en utilisant la base de données Google Scholar. On peut observer que les spillovers réciproques se manifestent à travers les citations de brevet, la mobilité de la main d'œuvre entre entreprises, l'apprentissage des formes d'organisation et de production, le partage des réseaux de distribution, etc. De même, on démontre que les spillovers réciproques surgissent grâce à la configuration de réseaux entre les multinationales et les acteurs de la région d'arrivée. On peut conclure que si bien que les spillovers réciproques sont difficiles à capturer et à mesurer, quand l'analyse se fait en fonction du processus de transfert de technologie c'est possible de faire le suivi de ce type d'externalités

Mots clef: spillovers, transfert de technologie, réseaux d’innovation, systèmes régionaux $d^{\prime}$ innovation, entreprises multinationales. 


\section{Una aproximación a la identificación, medición y generación de los spillovers recíprocos}

\section{Introducción}

La globalización de la economía ha generado una dinámica en la que las empresas multinacionales (EMN) no solo exportan tecnología a los países de acogida, sino que importan tecnología a través de las filiales donde se localizan (Dunning, 1993, 1998; Driffield y Love, 2003, 2005). Mansfield desde mediados de la década de 1980, ya advertía del proceso de transferencia de tecnología en ambos sentidos, e identificó un conjunto de externalidades que estimulaba este proceso y lo llamó spillovers o externalidades indirectas; estas pueden ser de dos clases: spillovers convencionales (SC) son aquellos beneficios que ganan las empresas locales por la presencia de las EMN; la segunda clase de externalidades son los spillovers recíprocos (SR) entendidos como las ganancias que las EMN logran por la interacción con los actores de las regiones donde localizan sus filiales (Mansfield, 1984).

Tradicionalmente a los spillovers se les han considerado como externalidades técnicas que surgen en gran medida por la utilización del conocimiento de agentes que se encuentran próximos e interdependientes (Cooke et al., 2004; Morgan, 2004). Se han usado para explicar el crecimiento endógeno (Romer, 1986, 1990), la aglomeración de firmas (Krugman, 1991) y el crecimiento de la firma (Dunning, 1993). No obstante su importancia y reconocimiento por teóricos tan influyentes, los spillovers no han estado libres de críticas. Breschi y Lissoni (2001), argumentan que los estudios econométricos no son contundentes en la medición de estas externalidades; Krugman (1993), aunque los reconoce, advierte que los spillovers no dejan rastro y no se pueden capturar con facilidad; Williamson (1989) argumenta que los costos de transacción son más relevantes que las externalidades tecnológicas en la comprensión del crecimiento de la firma.

El propósito de este trabajo es, a partir de una revisión de la literatura del proceso de transferencia inversa de tecnología (PITT), aportar a la comprensión de los SR como una clase particular de externalidades, con el fin de brindar elementos para la identificación, medición y efectos de estos beneficios indirectos en la localización de las EMN, aglomeración de firmas y la organización industrial. Los objetivos de este trabajo son establecer las diferentes clases de SR que surgen en el PITT, identificar algunos métodos y estrategias para acercarse a la representación y cuantificación de esta clase de externalidades y por último, identificar a las redes como fuentes de generación de spillovers recíprocos y convencionales.

En el documento se describen las cinco dimensiones que componen el proceso de trasferencia de tecnología, a continuación, se expone el método de análisis de citas seguido para selección de bibliografía pertinente para la identificación y medición de los SR. Se continúa con una discusión del papel de las redes en la transferencia de tecnología y la generación. Se finaliza con un conjunto de conclusiones.

\section{El proceso de transferencia inversa de tecnología (PITT)}

El PITT se lleva acabo en dos sentidos: el primero y más estudiado es la transferencia directa, que consiste en el derrame de conocimiento que hacen las EMN en los países y regiones de acogida (Cantwell, 1989). Con la globalización se ha puesto de manifiesto la transferencia inversa que se genera cuando la filial de una EMN adquiere conocimiento del país donde se localiza (Driffield y Love, 2003, 2005; Narula y Michel, 2009; Millar y Choi, 2009). Bozeman (2000) a partir de una revisión de las investigaciones sobre el proceso de transferencia de tecnología, identifica que sin importar el sentido, este proceso involucra 5 dimensiones.

a. La primera es la que estudia a los objetos de transferencia. Se ha identificado que los objetos en el PTIT suelen ser la generación de productos y procesos de alto, medio y bajo contenido tecnológico, la producción de patentes, licencias, planos, mapas y toda clase de conocimientos codificados, también se transfiere capital humano, estrategias de organización y modos de producción (Dunning, 1993, 1995, 1998, 2000).

b. La segunda dimensión es la que analiza a los agentes transmisores de tecnología que tradicionalmente se consideran como tal a 
las EMN. Una firma adquiere este estatus cuando a través de la inversión extranjera directa (IED) constituye una compañía o compra total o parcial de una empresa en un país distinto al de su casa matriz (Dunning, 1995; Cantwell y Mudambi, 2005; Schmidt y Sofka, 2009). En la era de la globalización, las EMN se están organizando en forma de red, donde el nodo principal es la casa matriz y alrededor del mundo localizan filiales diseñadas en función de los intereses que tengan en el país de acogida (Cantwell y Glac, 2004; Narula, 2009).

c. El tercer aspecto identificado por Bozeman como relevante en el PITT, es el análisis de las características de los agentes receptores de tecnología; entran en esta categoría los actores del país o región de llegada (Dunning, 1998). Con la internacionalización de la economía cada vez adquieren mayor relevancia las regiones como lugar de localización de filiales (Dunning, 1993; Krugman, 1991); porque debido a la cercanía de los actores, estas soportan el proceso de adquisición y generación de conocimiento en los llamados sistemas regionales de innovación (Cooke et al., 2004; Lundvall, 2002). La ventaja de las regiones se consolida si se configuran SRI eficientes y se estructuran redes donde se crea conocimiento, ocurren procesos de aprendizaje, se transfiere tecnología del exterior y facilita la difusión de las innovaciones (Lundvall, 2002; Edquist y Johnson, 1997).

d. El entorno es la cuarta dimensión a tener en cuenta en el PITT. Este aspecto lo constituye la política científica, tecnológica e industrial que define los incentivos en este proceso. En la era de la globalización un marco de análisis pertinente es el paradigma OLI (ownership, location, internalization) (Dunning, 2000). Aquí se analiza el impacto que tiene en la transferencia de tecnología, los cambios en la estructura de propiedad y generación de ventajas competitivas de las $E M N$, al igual que las modificaciones en las estrategias de localización de las filiales; por último se evalúa la transformación de la internalización de las actividades de las EMN (Dunning, 1993, 1998).

e. Por último pero no menos importante, está la dimensión de los medios y mecanismos de la PITT; entran en esta categoría los contratos de servicios técnicos, las compras de licencias, los convenios y toda clase de acuerdos que se establezcan para transferir conocimiento (Bozeman, 2000). Estos mecanismos son los que están permitiendo la formación de las redes de innovación y conocimiento, que cada vez son más relevantes para entender el PITT entre las filiales de las EMN y los actores de los sistemas regionales de innovación (Campbell y Carayannis, 2005).

Pero aunque en los mecanismos legales se reglamente el reparto de los beneficios, castigos y sanciones para cada una de las partes, debido a las propiedades de parcial exclusión en el corto plazo y no rivalidad en el largo plazo de la tecnología (Romer, 1990), la transferencia de conocimiento genera externalidades (Lundvall y Lorenz, 2010). Es en esta propiedad de la tecnología que surgen los SR y los SC (Narula, 2009); además, ha permitido que los agentes jueguen el doble papel de transmisores y receptores en el intercambio de conocimiento y tecnología. Esto explica el interés por entender el cómo se genera y cuantifica los SR.

\section{La selección de la literatura}

En este trabajo se aplicó el método bibliométrico de análisis de citas, con el fin de identificar la trayectoria que ha seguido el tema de los SR desde que Mansfield (1984) los propusiera como objeto de investigación. Se identificó que el estudio de los SR, está ligado con el tema de la transferencia de tecnología (Driffield y Love, 2003). Como se sabe, Bozeman (2000) identificó que este proceso lo componen 5 dimensiones: las características del agente transmisor, las del receptor, las características del objeto de conocimiento, los medios de transmisión y el entorno que determina la trasferencia.

Con base en estas dimensiones, se consultó la base de datos de Google Scholar y se seleccionaron los artículos que cumplieran con los siguientes criterios: que analizaran los SR en al menos dos de estas dimensiones y que fueran citados de manera recurrente por otros autores; de aquí que clasificaron como artículo representativo de la literatura, si aportaba conocimiento en los métodos de medición, generación e identificación de los SR, además, si profundizaba en cualquiera de los aspectos de la TPITT, y si tenía un acumulado creciente de citas según el tiempo. El resultado de la aplicación de los criterios se muestra en la Tabla 1. 


\begin{tabular}{|c|c|c|}
\hline \multicolumn{2}{|c|}{ Criterio de selección } & \# artículos \\
\hline \multicolumn{2}{|c|}{ Agente trasmisor } & 14 \\
\hline \multicolumn{2}{|c|}{ Agente receptor } & 30 \\
\hline \multicolumn{2}{|c|}{ Objeto de transferencia } & 21 \\
\hline \multicolumn{2}{|c|}{ Entorno de la transferencia } & 13 \\
\hline \multicolumn{2}{|c|}{ Medio de la transferencia } & 13 \\
\hline \multirow{6}{*}{ Tiempo } & $1984-1990$ & 6 \\
\hline & $1991-1995$ & 7 \\
\hline & $1996-2000$ & 4 \\
\hline & $2001-2005$ & 17 \\
\hline & $2006-2009$ & 10 \\
\hline & $2010-2012$ & 8 \\
\hline \multirow{6}{*}{$\begin{array}{l}\text { Impacto } \\
\text { (número de citas) }\end{array}$} & Más de 2000 & 23 \\
\hline & $2000-1501$ & 12 \\
\hline & $1500-1001$ & 8 \\
\hline & $1000-501$ & 3 \\
\hline & $500-101$ & 2 \\
\hline & $100-0$ & 4 \\
\hline
\end{tabular}

El análisis bibliométrico realizado a través de las citas, arroja que fueron analizadas 52 referencias de las cuales $67,3 \%$, fueron escritas desde el año 2000; esto implica que este es un tema que está cobrando importancia académica y existe un esfuerzo consiente por desarrollar métodos por identificar y analizar a los SR. Por otra parte, desde el análisis de citas, se observa que los SR son un tema relevante. Como se espera en el desarrollo de un tema científico, los artículos de mayor antigüedad tienen mayor número de citas y cada vez son menos los más citados, como se muestra en la Tabla 1, los 4 artículos con más de 2000 citas, datan de mediados de la década de 1980; por su parte, son más los artículos recientes y nuevos y como es lógico tienen un menor número de citas. Los 23 artículos escritos desde el año 2000 , el 44,2\% de las referencias tienen menos de 100 citas, lo cual indica que la producción científica sobre los SR es creciente en artículos y relevante en reputación.

Por otra parte, la tabla arroja que en términos de la trayectoria del análisis, la literatura se ha concentrado más en entender la generación de los SR, esto lo prueba el hecho que de las 52 referencias consultadas como relevantes en el tema, 30 se concentran en el análisis de las características del agente receptor, en este caso a las regiones como lugar de acogida y generadora de externalidades; mientras que 21 evalúan al objeto de transferencia como es el conocimiento y tecnología; finalmente se observa que en el estudio de los SR, el análisis se concentra en las EMN como agente transmisor y en este trabajo se identificaron 14 referencias; por último, se evidencia que el tratamiento del entorno y el de los medios de transmisión tiene igual relevancia, en tanto que se identificaron 13 artículos respectivamente.

\section{Los spillovers recíprocos}

Los SR se han hecho más evidentes con la globalización de la economía y están generando cambios en las organizaciones empresariales y gubernamentales (Driffield y Love, 2005). La presencia de los SR ha provocado que las EMN tengan que abrir las fronteras estableciendo redes con otras firmas y actores de los sistemas de innovación para acceder a conocimientos de otras empresas localizadas en otros países (Cantwell, 2009). Así mismo, los países y regiones establecen a los SR, como objetivo de política (Audresch y Feldman, 2006).

El canal a través del cual se lleva acabo el PTIT ha sido la IED (Dunning, 2000). Las fuentes de los SR son: el aprender haciendo, consistente en el proceso mediante el cual la EMN imita los métodos de organización e innovación de las empresas líderes del país anfitrión (Driffield y Love, 2003; Cantwell y Mudambi, 2005). La asociada a la aglomeración de firmas, en este caso, la empresa extranjera, busca aprovechar la calidad de los productos de los proveedores, la infraestructura de investigación y desarrollo del país de acogida (Driffield y Love, 2003; Lundvall y Lorens, 2010). De aquí se tienen tres clases de SR: los generados dentro de la misma industria de la EMN, los asociados a la relación con otros sectores de la industria, y los provenientes de fuentes externas (Driffield y Love, 2003, 2005).

Debido a la heterogeneidad de las fuentes, el cálculo y formas de los SR están siendo objeto de investigaciones (Mansfield, 1984; Driffield y Love, 2003, 2005; Schmidt y Sofka, 2009). Se pueden clasificar en cuatro vertientes los diferentes esfuerzos en el análisis de los SR.

Una primera investiga el problema de la localización de las filiales de las EMN con responsabilidad de generar $I+D$ desde la región de llegada; en esta línea están los trabajos de Athreye y Cantwell (2007), Driffield y Love (2003, 2005), Feinberg y Gupta (2004) y Cantwell y Mudambi (2005). Un segundo tipo de estudios se inscriben en los análisis de la concentración de la actividad innovadora y la generación de los SR, los trabajos de Jaffe et al. 
(1993), y Audretsch y Feldman (2006) son pioneros en esta línea de investigación.

Una tercera vertiente la integran trabajos como los de Molero (2000) y Narula (2009), Narula y Michel (2009); aquí de lo que se trata es de evidenciar la relación entre las multinacionales y los sistemas de innovación del país de acogida. Una cuarta vertiente la componen los trabajos que sitúan a las redes como mecanismo de transferencia de conocimiento y aprovechamiento de los SR; aquí son relevantes los trabajos de Campbell y Carayannis (2005), Paci y Batteta (2003), Cantner et al. (2008), Figueroa y Maggi (2011) y Costamagna (2011). Estos trabajos se han concentrado en explicitar que las redes son el mecanismo de organización más eficiente de transmisión de conocimiento entre los actores de las regiones de acogida y las filiales de las EMN.

\subsection{Spillovers recíprocos y su relación con la organización de la industria}

En la Tabla 2 se recoge una síntesis de los cinco trabajos más relevantes que mayor aporte e impacto han tenido en la explicación de los SR en la organización industrial y crecimiento de la firma.

La Tabla 2 muestra los trabajos más relevantes en la literatura de los SR en cuatro aspectos básicos, en cuanto a sus métodos empleados, la clase de spillover identificado, el hallazgo y la conclusión. Los anteriores elementos se describen en este apartado.

\begin{tabular}{|c|c|c|c|c|}
\hline Autor (es) y año & Método empleado & SR identificado & Hallazgo & Conclusión \\
\hline $\begin{array}{l}\text { Driffield y Love } \\
(2003)\end{array}$ & Econométrico & $\begin{array}{l}\text { Aprendizaje de las } \\
\text { MNS por imitación }\end{array}$ & $\begin{array}{c}\text { Existe mayor SR entre más I+D } \\
\text { haya en la región }\end{array}$ & $\begin{array}{l}\text { Las MNS modifican la } \\
\text { organización } \\
\text { industrial }\end{array}$ \\
\hline $\begin{array}{l}\text { Branstetter } \\
(2006)\end{array}$ & $\begin{array}{c}\text { Datos de panel } \\
\text { desbalanceados }\end{array}$ & Citas de patentes & $\begin{array}{l}\text { Hay una relación directa entre } \\
\text { la IED y las citas de patentes } \\
\text { en Japón y Estados Unidos }\end{array}$ & $\begin{array}{l}\text { Crecimiento de las } \\
\text { firmas MNS de ambos } \\
\text { países }\end{array}$ \\
\hline $\begin{array}{l}\text { Schmidt, Sofka } \\
\text { (2009) }\end{array}$ & $\begin{array}{l}\text { Probit y cálculo de } \\
\text { probabilidad }\end{array}$ & $\begin{array}{l}\text { La transferencia de } \\
\text { conocimiento de la } \\
\text { filiales a la casa } \\
\text { matriz }\end{array}$ & $\begin{array}{l}\text { La reputación, la legalidad, la } \\
\text { amenaza y el desequilibrio de } \\
\text { mercado afecta negativamente } \\
\text { la generación de SR }\end{array}$ & $\begin{array}{l}\text { Existen factores que } \\
\text { frenan el crecimiento } \\
\text { de la MNS }\end{array}$ \\
\hline $\begin{array}{l}\text { Yang, Mudambi, } \\
\text { Meyer (2008); } \\
\text { MillaryChoi(2009) }\end{array}$ & $\begin{array}{l}\text { Métodos cualitativos } \\
\text { (Encuesta: escala de } \\
\text { Liker) }\end{array}$ & $\begin{array}{l}\text { Conocimiento de } \\
\text { organización y } \\
\text { tecnológico }\end{array}$ & $\begin{array}{l}\text { Conocimiento que transfiere } \\
\text { desde la filial requiere de un } \\
\text { proceso de persuasión }\end{array}$ & $\begin{array}{l}\text { Los SR establecen } \\
\text { límites al crecimiento } \\
\text { de las MNS }\end{array}$ \\
\hline
\end{tabular}

Esta clase de spillovers se presentan cuando una EMN decide entrar a una economía porque existen líderes o campeones nacionales u otras multinacionales exitosas que poseen métodos de organización y tecnologías que pueden ser imitadas. En esta perspectiva es destacable el estudio de Driffield y Love (2003). A partir de un análisis de 21 sectores de la economía británica entre 1983 y 1992, muestran que la transferencia de tecnología inversa depende de las fuentes de aglomeración de la región y de la capacidad de absorción y aprendizaje de las EMNs en el sentido de Cohen y Levinthal (1989).

El resultado de los autores es relevante para la organización industrial del Reino Unido; por una parte, se tiene que existen externalidades tecnológicas de aglomeración para las empresas multinacionales que se localizan en sectores que invierten intensamente en
$I+D$, pero estas son menores para los sectores menos intensivos en I+D. Un segundo resultado es que las externalidades derivadas del aprendizaje tienen menor efecto para las EMN, que pertenecen a sectores de alto contenido tecnológico, mientras que son muy relevantes para los sectores de menor intensidad en gastos de I+D.

Con respecto a los spillovers recíprocos y el crecimiento de las EMN

Los estudios empíricos que se registran en esta dirección son múltiples, pero tienen como denominador común el análisis del crecimiento de las EMN mediante la IED.

Un primer grupo de trabajos se han concentrado en el análisis de las patentes, como fuente de SR, como instrumento de aprendizaje y difusión del conocimiento. Un estudio 
pionero situado en esta problemática que asoció las citas de las patentes como fuente de spillovers y la IED, es el llevado a cabo por Branstetter (2006). Aplicó la técnica de datos de panel desbalanceados a 189 filiales japonesas localizadas en EEUU y consultó citas de 2.368 patentes registradas entre los años 1980 y 1997.

El autor encontró suficiente evidencia estadística de que había difusión de conocimiento en ambas direcciones entre países, porque los inventores norteamericanos citan en alto porcentaje las patentes japonesas; por cada dólar de inversión japonesa en las filiales localizadas en EEUU que patentan, se incrementan positivamente las citas de los norteamericanos. Por otra parte, se muestra que los japoneses también aprenden de las patentes americanas; por cada dólar de IED japonesa en las filiales, las citas de los japoneses superan en el doble a las citas de los norteamericanos.

Un segundo grupo de estudios que se preocupan por identificar a los SR derivados de la presencia de las EMNs mediante la IED, se concentran en la evaluación de las relaciones entre las EMN con los actores del entorno local. En esta línea de investigación se destaca el estudio de Schmidt y Sofka (2009). El trabajo es relevante porque introduce el concepto de legitimidad y el de la responsabilidad de extranjería, para evaluar las relaciones de la EMN con los actores locales, a partir de un análisis de 1.129 empresas localizadas en Alemania, compuesta por filiales y empresas locales. El trabajo calcula la probabilidad que tiene una EMN de acceder a los conocimientos generados por las universidades, clientes y proveedores en las regiones alemanas.

Los autores obtuvieron los siguientes hallazgos: en primer lugar que la reputación, el cumplimiento de las leyes, las amenazas a las empresas locales y el temor al desequilibrio del mercado, se calculan como parámetros de probabilidad de trasferencia de conocimiento inversa. Que las filiales de las multinacionales tienen menor probabilidad de beneficiarse de la difusión del conocimiento generado en el entorno alemán que las empresas nacionales en el país de acogida. El otro hallazgo importante del trabajo es que la posibilidad de apropiación del conocimiento por parte de las EMN, está en relación inversa a lo tácito de éste en la producción; entre más codificado sea el saber de la industria, mayor aprovechamiento podrán hacer las EMN del entorno. Por lo anterior en comparación con las empresas locales, las multinacionales, tienen menor probabilidad de aprovechar los conocimientos de los clientes y universidades en el país de acogida.

Otro estudio que demostró la presencia de los SR derivados de la IED, fue el realizado por Yang et al., (2008); ellos introdujeron el concepto de la relevancia del conocimiento; en esta perspectiva, un conocimiento es relevante para la EMN, si este contribuye al incremento del valor de la empresa. Los autores encontraron que son cinco las clases de conocimientos de objeto de transferencia: los conocimientos tecnológicos, los asociados con las ventas y marketing, los de recursos financieros y los de gestión.

Dos son los hallazgos a relevar aquí: en primer lugar, que el conocimiento transferido de las EMN a las filiales, tiene el carácter de enseñanza y la transferencia opera por autoridad, mientras que el conocimiento que fluye de las filiales a la EMN, se transfiere después de un arduo proceso de persuasión. El segundo hallazgo, responde a los factores de transferencia: las características del país de acogida, la capacidad de adsorción de las filiales y de la EMN para asimilar conocimientos, son los principales determinantes del aprovechamiento de los SR.

En la misma perspectiva de transferencia de conocimiento, el trabajo de Millar y Choi (2009) muestra, al igual que el de Yang et al. (2008), que la transferencia de conocimiento de una filial a la casa matriz de una EMN, depende de la autoridad, pero además este proceso tiene varias barreras u obstáculos, los autores identificaron como los más relevantes: el contrato psicológico, la desconfianza en la justicia, la falta de motivación intrínseca, la distancia psíquica y la responsabilidad de extranjería.

\subsection{Los spillovers geográficos y la localización de las EMN}

La Tabla 3 muestran a manera de síntesis los cinco trabajos que han realizado un aporte significativo en la identificación y medición de los SR derivados de la localización de las filiales. 
La Tabla 3 muestra los trabajos más relevantes en la literatura de los SR en cuatro aspectos básicos: los métodos empleados, la clase de spillover identificado, el hallazgo y la conclusión. Los anteriores elementos se describen en este apartado.

\begin{tabular}{|c|c|c|c|c|}
\hline Autor (es) y año & Método empleado & SR identificado & Hallazgo & Conclusión \\
\hline $\begin{array}{l}\text { affe., } \\
\text { Henderson y } \\
\text { Trajtemberg } \\
\text { 1993) }\end{array}$ & $\begin{array}{l}\text { Probit de cálculo de } \\
\text { probabilidad de que } \\
\text { una patente sea } \\
\text { citada }\end{array}$ & Citas de patentes & $\begin{array}{l}\text { Existe una mayor } \\
\text { probabilidad de que una } \\
\text { patente sea citada en la } \\
\text { región de origen que en } \\
\text { cualquier otra parte del } \\
\text { mundo }\end{array}$ & $\begin{array}{l}\text { Mayor concentración } \\
\text { dentro de la región que } \\
\text { patenta }\end{array}$ \\
\hline $\begin{array}{l}\text { Feinberg y Gupta } \\
(2004)\end{array}$ & $\begin{array}{l}\text { Probit de cálculo de } \\
\text { probabilidad de que } \\
\text { a una filial se le } \\
\text { asignen } \\
\text { responsabilidad de } \\
\text { desarrollos } \\
\text { tecnológicos }\end{array}$ & $\begin{array}{l}\text { Conocimiento } \\
\text { científico de la } \\
\text { región de acogida }\end{array}$ & $\begin{array}{l}\text { Una filial tendrá mayor } \\
\text { probabilidad de que le } \\
\text { asignen tareas de } \\
\text { desarrollo de conocimiento } \\
\text { entre mayor sea la } \\
\text { inversión en I+D que realice } \\
\text { la región de acogida }\end{array}$ & $\begin{array}{l}\text { La casa matriz delegará } \\
\text { más responsabilidad de } \\
\text { generación de } \\
\text { investigación a las filiales } \\
\text { que se localicen en } \\
\text { regiones intensivas en } \\
\text { conocimiento }\end{array}$ \\
\hline $\begin{array}{l}\text { Alonso, } \\
\text { Chamorro y } \\
\text { González (2004) }\end{array}$ & $\begin{array}{l}\text { Índices: Gini, Maurel } \\
\text { y Sédillot; Ellison y } \\
\text { Glaeser }\end{array}$ & $\begin{array}{l}\text { Spillovers derivados } \\
\text { de la concentración } \\
\text { industrial }\end{array}$ & $\begin{array}{l}\text { Existe una relación } \\
\text { directa entre la } \\
\text { localización filiales y la } \\
\text { calidad de los } \\
\text { proveedores }\end{array}$ & $\begin{array}{l}\text { Las regiones se esforzarán } \\
\text { más por atraer inversión } \\
\text { extranjera entre mayor } \\
\text { concentración industrial } \\
\text { tenga }\end{array}$ \\
\hline
\end{tabular}

El análisis de esta clase de spillovers, parten del reconocimiento que en el PITT opera un triángulo en el que las EMN participan en dos vértices, la casa matriz y el segundo es la filial localizada en una región determinada; el tercer vértice lo ocupa el o los actores del sistema regional de innovación donde se localiza la filial (Narula y Michael, 2009). Como se observa, la filial es el agente que actúa como paso comunicante a través del cual circula el conocimiento tanto de la EMN a la región de acogida como desde ésta a la casa matriz de la multinacional (Feinberg y Gupta, 2004). Los estudios basados en este triángulo se clasifican en tres grupos: los que estudian el proceso de localización de las filiales, los que analizan las características del territorio como fuente de generación de spillovers para las empresas en general y las EMN en particular; por último están los estudios que evalúan la lógica de interacción de las EMN con el entorno o sistema de innovación.

\section{La búsqueda de spillovers de las EMN mediante la localización de las filiales}

Las investigaciones que se ocupan de este tema han evidenciado que las EMN localizarán filiales en función de la estrategia de internacionalización y que existe una estrategia particular si el objetivo es explotar mercados locales, mientras que otra será la estrategia si el objetivo es crear competencias y aprovechar spillovers (Cantwell y Mudambi, 2005; Cantwell y Glac, 2004; y Guimón, 2008).
Un estudio que prueba esta hipótesis general, fue realizado por Feinberg y Gupta (2004), a partir de un análisis de los datos en el periodo comprendido entre 1989 a 1996, a 2.306 filiales pertenecientes a 361 multinacionales con la casa matriz en EEUU, localizadas en 10 industrias de 11 países de la OCDE. Los autores calcularon la probabilidad que una EMN asigne responsabilidades de realizar proyectos de I+D a una filial del total del conjunto que poseen en el resto del mundo; los hallazgos fueron:

La probabilidad que se asigne responsabilidades de I+D, a una filial, está relacionada positivamente con la magnitud de los gastos de investigación y desarrollo que realicen las empresas de la región de acogida. Asi mismo, se obtuvo que el que una filial se designe como responsable de I+D está en relación negativa con la relación entre la dispersión y la magnitud de los gastos en I+D. Igualmente la confianza de una EMN en una filial para ejecutar investigaciones, está en relación directa con la capacidad de la multinacional de abastecer un mercado local y global. De la misma forma se mostró que entre más participación en la propiedad de la filial tenga la EMN, mayor será la probabilidad de que la filial emprenda proyectos de investigación a nombre de la EMN. En cuanto las características de la EMN, se encontró que la elección de una filial para ejecutar actividades de I+D, depende por una parte del comercio intra-firma de la EMN y por otra del número de filiales que posea la EMN. 


\section{Los spillovers geográficos}

Las EMN, también suelen capturar externalidades derivadas de la cercanía de industrias relacionadas. Los estudios que investigan esta clase de efectos, se concentran en dos aspectos articulados, las externalidades que se derivan de la aglomeración de empresas o formación de clusters y la concentración de la actividad innovadora. A la suma de los dos tipos de spillovers se les conoce como spillovers geográficos (Morgan, 2004).

El tema de los spillovers asociados a la localización se remonta a los estudios de Jaffe et al. (1993). Para el análisis de esta clase de spillovers, los autores se fundamentan en las tres premisas de Marschall y luego retomadas por Krugman, para explicar la concentración geográfica de la producción; en estas se afirma, que la concentración industrial se debe a tres factores: a la existencia de una mano de obra calificada, a la configuración de un mercado de bienes intermedios y a la generación de una osmosis tecnológica o externalidades (Krugman, 1993). Este autor ha advertido que los dos primeros factores son medibles, mientras que este último no es fácil de rastrear.

Jaffe et al. (1993), intentan allanar esta dificultad, mostrando como alternativa las citas de las patentes como evidencia del flujo de conocimiento y analizan las patentes de las universidades y empresas de los estados de los EEUU, divididas en dos cohortes, un grupo de 1975 y otro de 1980. Aplican un modelo Probit a cada grupo de patentes, para calcular la probabilidad que una patente de un inventor de un estado particular de la unión, sea citada por otro inventor localizado en el mismo estado. Ellos encuentran que existe suficiente evidencia estadística para aceptar la hipótesis que las patentes tienden a estar geográficamente localizadas, es decir, la probabilidad de que una patente asignada a un inventor de un estado sea citada por otro inventor del mismo estado, es mayor que la probabilidad que la patente sea citada en otro estado o en el extranjero.

Krugman (1991) y los hallazgos de Jaffe et al. (1993), plantearon el problema de cuál debería ser la unidad geográfica de análisis de una función de producción de conocimiento. Para avanzar en este debate, Audretsch y Feldman (2006), analizan la relación entre la difusión del conocimiento y el espacio regional. A partir de una revisión de la actividad económica y patentadora de los estados de los EEUU, muestran que lo que es cierto para la producción, es más evidente para la actividad innovadora, es decir, existe una concentración geográfica para la innovación y difusión del conocimiento; estos autores, identifican un conjunto de factores que explican la importancia de las regiones como fuente de spillovers: los Spillovers se presentan más donde existe competencia y cooperación, capital humano y universidades de calidad, capital de riesgo, y espíritu empresarial.

En la línea de los spillovers derivados de la aglomeración y las externalidades inter-industrias, vale la pena resaltar el trabajo de Alonso et al. (2004); los autores se proponen evidenciar la presencia de spillovers geográficos y sectoriales de la industria en España; para ello construyen tres índices: el Índice de Gini, el de Maurel y Sédillot, y el de Ellison y Glaeser. A partir de estos muestran que en España existe concentración industrial en 5 regiones: la Comunidad de Madrid, Catalunya, País Vasco, Andalucía y Valencia; en estas se concentra gran parte de la actividad económica española; de igual manera se muestra que la producción se concentra en 15 sectores del total de los sectores industriales.

La relevancia de este estudio es múltiple. Los autores evidencian que la probabilidad que dos plantas se localicen en una misma región y, una vez elegida la región, se localicen en una misma provincia, está determinada por los spillovers de la región, más los spillovers de la provincia, más el producto de ambos spillovers. A partir de este hallazgo, los autores concluyen que habrá una competencia entre regiones por ofrecer las mejores condiciones para atraer la inversión extranjera y a su vez, habrá competencia entre provincias de una región para atraer a las firmas multinacionales.

\section{La formación de redes como estrategia de captura de los spillovers de las EMN y las regiones}

Una red de conocimiento es una variante de las redes sociales, pero que a diferencia de estas redes, lo que circula es conocimiento y tienen la función de permitir que este llegue a los actores que pertenezcan a la red (Verspagen, 1993; Ernst y Kim, 2002; Campbell y Carayannis, 2005; Cantner et al., 2008; Figueroa y Maggi, 2011; Costamagna, 2011). Las redes de innovación están compuestas por un conjunto de organizaciones; cuando estas tienen el objetivo de aprovechar los spillovers recíprocos y convencionales, en la red es posible distinguir dos clases de actores: uno lo constituyen las EMN, y las filiales; el otro grupo de actores se agrupa en el sistema de innovación, integrado por universidades, empresas locales, institutos de investigación, Gobierno y organismos de apoyo (Verspagen, 1993; Edquist y Johnson, 1997; 
Molero, 2000; Ernst y Kim, 2002; Narula y Michel, 2009; Pietrobelli y Rabellotti, 2011).

El estudio de Gertler y Levitte (2003), es digno de presentar en esta perspectiva; muestra que en el sector de la biotecnología, las empresas canadienses ocupaban para ese año el tercer puesto en capacidad innovadora con más de 2000 patentes. Se muestra que el éxito competitivo de las empresas está asociado con la capacidad de las firmas a integrarse en redes de innovación mundiales y en concentrarse en clusters regionales de innovación. Los hallazgos empíricos, muestran que en la biotecnología había una tendencia hacia la integración de las empresas canadienses con redes mundiales de comercio, ya sea para la comercialización de las patentes como para la búsqueda de trabajadores calificados. Por su parte, la integración local, está en función de asociaciones para lograr financiarse mediante capital de riesgo.

Debido a la importancia de las redes de innovación y conocimiento como fuente de los SR y SC, a continuación se describe cómo cada relación de red es una potencial fuente de spillovers.

1․ . Joint Ventures para emprender proyectos de investigación universidad-empresa. Estos acuerdos generan dos clases de spillovers: el primer y más obvio resultado son los spillovers que se generan gracias a la movilidad de estudiantes y profesores que se vinculan a la EMN; este recurso humano aprende de esta clase de empresas y luego aplican en las empresas locales; esta externalidad opera en los sectores intensivos en aprendizaje del tipo Learning bi doing (Lundvall y Lorenz, 2010; Harmaakorpi y Melvas, 2004). Por otra parte, las EMN ganan en conocimiento en este tipo de acuerdos cuando las filiales logran apropiarse del conocimiento generado en las universidades localizadas en la región y se evidencia cuando las EMN patentan en el país de acogida (Criscuolo, 2003).

2․ Acuerdos multilaterales en $I+D$ entre la universidad empresa y el Estado. Estos vínculos pueden ser entre otros la constitución de consorcios de investigación, conformación de infraestructuras de innovación del tipo parques científicos, bancos de datos, plataformas virtuales (Koschatzky, 2002). Los acuerdos de esta naturaleza, contribuyen a la capacidad de adsorción de la región en el sentido de Cohen y Levinthal (1989), Simmie (2003), Cantner et al., (2008); entonces las EMN, se localizan en las regiones que siguen esta estrategia, cuando pueden aprovechar conocimiento público e infraestructuras de investigación e innovación (Cantwell y Glac, 2004). Como fruto de estos vínculos se generan tan- to SC como SR; los primeros se evidencian cuando las EMN, instauran laboratorios de investigación y plantas de producción de partes del producto final; el SRI de la región se beneficia con la llegada de conocimientos en ingeniería y administración (Lundvall y Lorenz, 2010). Por su parte la EMN, gana las externalidades que genera el capital humano formado en la región (Audretsch y Feldman, 2006).

$3^{0}$. Relaciones interempresariales para el intercambio de conocimiento y tecnología. Entran en esta categoría los acuerdos de licencia y second sourcing y procesos de subcontratación, las redes de proveedores, el intercambio tecnológico y científico orientados hacia la cadena de valor, acuerdos informales de transferencia de conocimiento (Freeman, 1991; Koschatzky, 2002). Este tipo de convenios suelen ser los más recurrentes entre las filiales de las EMN y las empresas locales. Los efectos colaterales derivados son múltiples, como se mostró antes. Entre las empresas puede haber intercambio de conocimiento, pero según la literatura, el recibido por la EMN debe ser pertinente y relevante, deberá ser complementario e incrementar el valor de la EMN, mientras que el conocimiento que recibe la empresa local lo recibe mediante autoridad (Yang et al., 2008).

De nuevo en este tipo de acuerdos se generan spillovers convencionales y recíprocos; las empresas locales ganan a través del incremento de la productividad derivada de la adquisición de tecnología derramada por la multinacional, se distinguen múltiples maneras de capturar externalidades, las formas de organización de la producción y apertura de canales de distribución suelen ser las ganancias más recurrentes que deja la presencia de una multinacional en la región, esto ocurre sobre todo cuando las empresas locales logran integrarse en las cadenas globales de valor de las cuales la multinacional hacen parte (Pietrobelli y Rabelloti, 2011). Por su parte las EMN ganan externalidades mediante acuerdos con empresas locales, cuando estas son líderes en el país de acogida y les facilita entrar en los mercados locales, aprovechar los incentivos que en materia de investigación y desarrollo se dispongan en la región (Cantwell, 1989; Feinberg y Gupta, 2004).

$4^{\circ}$. Convenios para realizar inversiones directas inducidas por la tecnología. Esta forma de articulación se da entre las EMN y las regiones de acogida, este proceso requiere que ambas partes tengan ventajas competitivas y capacidades para permitirse crear un centro de alta tecnología (Branstetter, 2006; y Vázquez 2011). Este tipo de relaciones es el que está permitiendo la creación de centros de excelencia en tecnologías e industrias especializadas 
en países de economías emergentes como Brasil, India y China (Narula, 2009). Este tipo de acuerdos, es una potencial fuente de generación de spillovers convencionales y recíprocos.

Por una parte las multinacionales que deciden constituir un centro de excelencia en una región, lo hacen buscando reducción de costos, incentivos tributarios, mano de obra calificada, experiencia en investigación y desarrollo, etc. (Athreye y Cantwell, 2007). Por otra parte, cuando una región logra atraer un centro de excelencia o una instalación científica de una multinacional, el sistema de innovación tiene un impacto positivo; en primer lugar se desencadena un círculo virtuoso de desarrollo en la medida que el solo hecho que llegue este tipo de inversión hará que la región se vuelva más atractiva para otros inversionistas, los actores locales se incentivan para invertir en capital humano, capital de riesgo y mejorará probablemente el espíritu empresarial (Heijs, 2010; Vázquez, 2011).

\section{Conclusiones}

En este trabajo se ha logrado, a través de una revisión de la literatura, identificar a los SR. Las maneras de analizar este tipo de spillovers son las citas de patentes, la movilidad de la mano de obra, el aprendizaje de las formas de organización y producción y los canales de distribución. La revisión de la literatura ha dejado conceptos tales como: el de responsabilidad de extranjería, el conocimiento relevante, las barreras legales, la formación de redes de proveedores y el aprendizaje por imitación, entre otros; y se ha evidenciado que tienen un impacto significativo en temas como el crecimiento de la firma, la localización de las filiales y la organización industrial; de aquí que los SR deben ser tenidos en cuenta para el diseño de política industrial y científica.

Una conclusión apenas comprensible es que si bien esta clase de externalidades no es fácil de capturar y de medir, su dificultad radica en la naturaleza intangible del conocimiento que no siempre es sensible de ser contenido en cosas y objetos, porque está presente en las personas, ideas, representaciones y toda clase de formas no siempre tangibles.

Los trabajos aquí presentados como ejemplos para capturar a los SR, dejan como enseñanza metodológica que para capturar a los spillovers se requiere combinar metodológicamente varios marcos de análisis. Desde lo teórico es prudente combinar el análisis de redes con el de transferencia de tecnología y para realizar análisis empíricos de los estudios evaluados se concluye que se deben com- binar métodos cuantitativos y cualitativos, uso de instrumentos y tratamiento de datos con creatividad. Esta combinación de metodologías hace del tema sensible a críticas, sin embargo, es un tema que pese a ser relativamente joven, ha logrado consolidarse como relevante.

Los estudios muestran que las redes son las fuentes de generación y explotación de los spillovers; así que los vínculos y acuerdos encaminados a la formación de infraestructuras, intercambios de colectivos de investigación, los acuerdos multilaterales y los demás aquí planteados, permiten inferir que la relación entre EMN y SRI es una dinámica necesaria en la era de la globalización; por tanto, entender el análisis de las redes, sus propiedades de centralidad e intermediación, la lógica de los vínculos, el papel de la distancia y el estímulo a la asociación, podrá permitir diseñar políticas amplias, incluyentes, globales y colaborativas.

\section{Referencias}

Alonso, V.O. Chamorro, J.M. y González, X. (2004). Agglomeration economies in manufacturing industries: the case of Spain. Applied Economics, 36 (18) (pp. 2103-2116).

Athreye, S. y Cantwell, J. (2007). Creating competition? Globalisation and the emergence of new technology producers. Research Policy, 36 (pp. 209-226).

Audretsch, D.B., Feldman, M.P. (2004). Knowledge spillovers and the geography of innovation in Henderson, J.V., Thisse, J.F. (Eds.). Handbook of regional and urban economics (vol. 4). Amsterdam, North Holland: Elsevier.

Bozeman, B. (2000) Technology transfer and public policy: a review of research and theory. Research Policy, 29 (pp. 627-655).

Branstetter, L. (2006). Is foreign direct investment a channel of knowledge spillovers? Evidence from Japan's FDI in the United States. Journal of International Economics, 68 (pp. 325-344).

Breschi, S. y Lissoni, F. (2001) Knowledge spillovers and local innovation systems: a critical survey. LIUC Papers in Economics, 84 (pp 1-30).

Campbell, D. Carayannis, E. (2005). Knowledge creation, diffusion, and use in innovation networks and knowledge clusters, a comparative systems approach across the United States, Europe and 
Asia. Westport, USA, Londres, UK: Praeger (pp. 67-100).

Cantner, U, Meder, A. \& Wal, A. (2008). Innovator networks and regional knowledge base. JENA Economic Research Papers, 42 (pp. 1-34).

Cantwell, J. (1989). Technological innovation and multinational corporations. Oxford, UK: Basil Blackwell.

Cantwell, J. (2009). Blurred boundaries between firms, and new boundaries within (large multinational) firms: the impact of decentralized networks for innovation. Seminar Presentation. Maastricht, Nederland, August, 2008. UNU, MERIT.

Cantwell, J. y Glac, K. (2004). La estrategia tecnológica de las empresas multinacionales y el desarrollo de capacidades tecnológicas locales. Cuadernos de economía y dirección de la empresa, 20 (pp. 83-102).

Cantwell, J. y Mudambi, R. (2005). MNE competencecreating subsidiary mandates. Strategic Management Journal, 26 (pp. 1109-1128).

Cohen, W, Levinthal, D. (1989). Innovation and learning: The two faces of R\&D. The economic journal, 99 (pp. 569-596).

Cooke, P. Heidenreich, M. \& Braczyc, H.J. (2004) Regional Innovation Systems: The role of governance in a globalized world. Londres, UK: Editorial Routledge.

Costamagna, P. (2011). De los servicios empresariales a las redes para la innovación del territorio. En: Curbelo J.L., Parrilli M.D. y Alburquerque, F. (Ed.). territorios innovadores y competitivos. Madrid, España: Marcial Pons Ediciones (219-236).

Criscuolo. P, (2003). Reverse Technology Transfer: A Patent Citation Analysis of the European Chemical and Pharmaceutical sectors. Brighton, UK: SPRU Electronic Working Paper Series (pp. 17. 35).

Driffield, N. y Love, J. (2003). Foreign direct investment, technology sourcing and reverse spillovers. The Manchester School, 71, (6) (pp. 659-672).

Driffield, N. y Love, J., (2005). Who gains from whom? spillovers, competition and technology sourcing in the foreign-owned sector of UK manufacturing. Scottish Journal of Political Economy, 52, (5) (pp. 663-686).
Dunning, J.H. (1993).Challenges for theorizing about MNEs and MEN activity. En: The globalization of business. Londres, UK: Routledge (37-167).

Dunning, J.H. (1995). Reappraising the eclectic paradigm in an age of alliance capitalism. Journal of International Business Studies, 26 (pp. 461-491).

Dunning, J.H. (1998). Location and the multinational enterprise: A neglected factor? Journal of international Business Studies, 29 (1) (pp. 45-66).

Dunning, J.H. (2000). The eclectic paradigm as an envelope for economic and business theories of MNE activity. International Business Review, 9 (pp. 163-190).

Edquist, C. y Johnson B. (1997). Institutions and organizations in systems of innovation. En Edquist, C. (Ed.). Systems of Innovation: technologies, institutions and organizations. Londres, UK: Pinter Publishers (pp. 41-63).

Elsinore, D., Guimón, J. (2008). Government strategies to attract R\&D-intensive FDI. The journal of Technology Transfer, 34 (pp. 364-379).

Ernst, D. y Kim, L. (2002). Global production networks, information technology and knowledge diffusion. Industry \& Innovation, 9 (3) (pp. 147-153).

Feinberg, S. y Gupta, A. (2004). Knowledge spillovers and the assignment of r\&d responsibilities to foreign subsidiaries. Strategic Management Journal, 25 (pp. 823-845).

Figueroa, C. y Maggi, C. (2011). Redes presenciales y virtuales para la innovación. En: Curbelo J.L. Parrilli M.D. y Alburquerque, F. (Ed.) territorios innovadores y competitivos. Madrid, España: Marcial Pons Ediciones (pp. 195-218).

Freeman, C. (1991). Networks of innovations: A synthesis. Research Policy, 20 (p. 499).

Gertler, M. \& Leavitte, Y. (2003). Local nodes in global networks: The geography of knowledge flows in biotechnology innovation. Conferencia DRUID Summer. Copenhagen, Dinamarca, Junio 2003, DRUID.

Harmaakorpi, V. y Melvas, H. (2004). Data, information and knowledge in regional innovation networks Quality considerations and brokerage functions. European Journal of Innovation Management, 11 (1) (pp. 103-124). 
Heijs, J. (2010) Política tecnológica, aprendizaje y capacidad de absorción de conocimientos: los círculos viciosos y virtuosos. En: Parrilli, M.D. (Ed.) innovación y aprendizaje: lecciones para el diseño de política. Zamudio, España: Innobasque (pp. 324-351).

Hekkert, M.P., Suurs, R.A.A., Negro, S.O., Kuhlmann, S. \& Smits, R.E.H.M. (2007). Functions of innovation systems: A new approach for analysing technological change. Technological Forecasting \& Social Change, 74 (pp. 413-432).

Jaffe, A., Henderson, R. \& Trajtemberg, M. (1993). Georgraphic localization of knowledge spillovers as evidenced by patent citations. The Quarterly Journal of economics, 108 (3) (pp. 577-598).

Koschatzky, K. (2002). Fundamentos de la economía de redes. Especial enfoque a la innovación. Revista Economía Industrial, 346 (pp. 15-26).

Krugman, P. (1991). Increasing returns and economic geography. Journal of Political Economy, 99 (3) (pp. 483-499).

Krugman, P. (1993). Geografía y comercio. Barcelona, España: Antoni Bosch Editor.

Lundvall, B. (2002). Estados-nación, capital social y desarrollo económico. Un enfoque sistémico de la creación de conocimiento y el aprendizaje en la economía global. Revista de Economía Mundial, 7 (pp. 69-90).

Lundvall, B. y Lorenz, E. (2010). Innovación y desarrollo de competencias en la economía del aprendizaje. Implicaciones para las políticas de innovación. En: Parrilli, M.D. (Ed.), innovación y aprendizaje: lecciones para el diseño de políticas. Zamudio, España: Innobasque (pp. 44-101).

Mansfield, E. (1984). R\&D and innovation: Some empirical findings. En: Griliches, Z. (Ed.) R\&D patents and productivity. Chicago, USA: University of Chicago Press (pp. 127-154).

Millar, C. \& Choi, C. (2009). Reverse knowledge and technology transfer: imbalances caused by cognitive barriers in asymmetric relationships. International Journal of Technology Management, 48 (3) (pp. 389-402).

Molero, J. (2000). Las empresas multinacionales y el sistema español de innovación. En: Molero, J. Competencia global y cambio tecnológico: un desafío para la economía española. Madrid, España: Piramide (pp. 251-288).

Morgan, K. (2004). The exaggerated death of geography: learning, proximity and territorial innovation systems. Journal of Economic Geography, 4 (pp. 3-21).
Narula, R. (2010). Much ado about nothing, or sirens of a brave new world?: MNE activity from developing countries and its significance for development. París, Francia: United Nations University (UNU), Maastricht Economic and Social Research and Training Centre on Innovation and Technology (MERIT).

Narula, R. \& Michel, J. (2009). Reverse knowledge transfer and its implications for European policy. Maastricht, Nederland: Maastricht Economic and social Research and training centre on Innovation and Technology MERIT).

Paci, R. \& Batteta, E. (2003). Innovation networks and knowledge flows across the european regions. Working Paper No. 2003_13. Cagliari - Italy: Centre For North South Economic Research (CRENOS), (pp. 1-14).

Pietrobelli, C. y Rabelliotti, R. (2011). Cadenas de valor globales y sistemas de Innovación: ¿oportunidades de aprendizaje para los países en desarrollo? En: Curbelo, J.L., Parrilli, M. y Alburquerque, F. (Ed.), Territorios innovadores y competitivos. Madrid, España: Marcial Pons Ediciones (pp. 75-88).

Romer, P. (1986). Increasing returns and long-run growth. The Journal of Political Economy, 94 (5) (pp. 1002-1037).

Romer, P. (1990). Are nonconvexities important for understanding growth? The American Economic Review, 80 (2) (pp. 97-103).

Schmidt, T, Sofka, W. (2009). Liability of foreignness as a barrier to knowledge spillovers: Lost in translation? Journal of International Management, 15 (pp.460-474).

Simmie, J. (2003). Innovation and urban regions as national and international nodes for the transfer and sharing of knowledge. Regional studies, 37 (6-7) (pp. 607-620).

Vázquez, A. (2011). Los territorios innovadores, espacios estratégicos del desarrollo. En: Curbelo J.L., Parrilli, M. y Alburquerque, F. (Ed.) Territorios Innovadores y Competitivos. Madrid, España: Marcial Pons Ediciones (pp. 75-88).

Verspagen, H. (1993). Uneven growth between interdependent economies: an evolutionary view on technology gaps, trade and growth. Maastricht, Nederland: Universitaire Pers Maastricht.

Yang, Q., Mudambi, R. \& Meyer, C. (2008). Conventional and reverse knowledge flows in multinational corporations. Journal of Management, 34 (5) (pp. 882-902).

Williamson, O. (1989) Las instituciones económicas del capitalismo. Ciudad de México, México: Fondo de Cultura Económica. 